\title{
Reconstruction of Ancient Two-Dimensional Objects
}

\author{
Md Jan Nordin and Nada A. Rasheed
}

\begin{abstract}
This work proposes a method for reconstructing ancient objects based on the use of 2D images. The method exploits the features of the edges of fragments, as well as the colors near the edges of the fragments. The main idea of this method is to obtain accurate contours of fragments, and consider them as curves. The next step is to apply a polynomial function to compute the coefficients of the curve, which is effective to recognize other edges of corresponding fragments. Moreover, extracting color features near the edges of fragments particularly effective in matching the pair of fragments. In order to recognize the corresponding edges of the other fragment, the correlation coefficient and the highest intersection of colors were used. This method achieved a high success rate by relying on the coefficients of a polynomial function, and the intersection of colors between pairs of edges of the fragments.
\end{abstract}

Keywords - archaeological fragments, reconstruction objects, polynomial function.

\section{INTRODUCTION}

The problem of the reconstruction of archaeological fragments is an important challenge in the pattern recognition field. Thus, it is greatly applied in archaeology, because of the high value of these fragments to scholars. It also helps archaeologists to make inferences about past civilizations [1][2][3]. Therefore, it is necessary to propose methods for the reconstruction of the archaeological objects before they are lost or damaged [4]. Hence, numerous authors attempted to find solutions to the problem of reconstructing objects from a collections of fragments via proposed methods, rather than assembling them manually, which is time intensive and tedious [5].

A study by [6] adopted the shape information of pottery fragments for the reconstruction of the irregular fragments; they assumed the surfaces of objects are smooth, and the fracture lines are curves without a width. A system to reconstruct the earthenware was provided by [7]. Their method is based on the color patterns and similarity of the shape. The basis of their method depends on detecting the contours of the fragments. The approach presented by [8] depends on extracting the silhouette contours from each fragment. Through exploiting the thematic content of the fragments, such as the alternate and parallel black and blue stripes, the researchers [9] presented an approach for reconstructing the wall paintings of a Greek island. The work by [10] improved the previous work of the reconstruction of the wall paintings

Center for Artificial Intelligence Technology, Faculty of Information Science and Technology, Universiti Kebangsaan Malaysia and floor fragments. They relied on contours and chromatic content near the edge of the fragments, as well as the thematic content which was traced across borders of the fragments. The main objective of this work is to propose a method to reconstruct archaeological objects accurately.

\section{MATERIALS AND METHODS}

The proposed method comprises several sets of procedures; the first step consists of obtaining the 2D images of the fragments. The next step involves applying an edge filtering procedure. A polynomial function is then applied to obtain the coefficient values. Finally, the matching process is conducted between similar pair edges of different fragments based on the values of the coefficients, and the intersection of colors.

\section{A. Images acquisition}

In order to apply the proposed algorithm, six images of the fragments were obtained by loading into memory. The images include the fragments of pots, vases, bowls, and water jugs. Each image is in .JPG format, and has dimensions of $300 \times$ 210 pixels.

\section{B. Edge detection}

The task of extracting features means obtaining the most relevant information from the original data, and representing them in the lower dimensional space. Thus, the method depends on the edges extracted from the images of the fragments. This work relies on commands from MATLAB 2014a, and an algorithm for accurately detecting the edges of the fragments was applied. In this case, we obtained the coordinates for each point of the edges of the fragment, so each of the contours is divided into several curves adopting on the joint of the contour (salient points). In order to approximate the variation between points of the curve, we applied a standard score that is represented by the following Equation [11].

$$
z=\left(x_{i}-\mu\right) / \sigma
$$

Where xi represents the original point value; $\mu$ and $\sigma$ represent the means and standard deviation respectively.

\section{Apply a polynomial function}

Considered a polynomial function as a smooth curve without breaks or corners. An example of a polynomial function is a quadratic or a cubic, among others. The general form can represented as in Equation 2.

$$
f(x)=a_{n} x^{n}+a_{n-1} x^{n-1}+\ldots \ldots+a_{2} x^{2}+a_{1} x+a_{0}
$$


Where $x$ is a variable and the real numbers of the coefficients $a_{n}, a_{n-1}, \ldots, a_{2}, a_{1}, a_{0}$ are constants; the positive integer $n$ represents the degree of the formula, and is not equal zero [12].

In order to calculate the coefficients and find polynomial equations for each curve, we applied an algorithm that is similar to the one used in [13], which adapted the model of the $6^{\text {th }}$ degree. Therefore, in this stage, we accomplished and obtained all coefficients for every sub-contour, and defined all functions for each edge of the fragments stored, mainly for use in the next step.

\section{Color extraction}

In order to reduce the number of potential matches, this study relies on the colors near the edges of the fragment. Considering the colors of the fragment is an important factor that should be taken into account to reconstruct the objects. Since the reconstruction of the objects depends on the edges of the fragments, this work used the color of the edges, without the entire surface. Hence, this surface will be represented as a strip that surrounds the edge of the fragment, where the width of the strip is 10 pixels. The strip of the edge can be obtained through applying the next algorithm.

\section{Step1: D = read (image)}

Step2: Separated matrix colors into three matrices (Red, Green, Blue).

$$
\begin{aligned}
& \text { Step3: Ed = Edges extraction } \\
& \begin{array}{l}
{[N, M]=\operatorname{size}(D)} \\
B=\text { zeros matrix } \operatorname{size}(N, M) \\
\text { For } i=1: N-10 \\
\text { For } j=1: M-10 \\
\text { If } E d(i, j)==0 \\
B(i, j)=D(i, j) \% \text { Return the original value of pixels } \\
\text { End if }
\end{array}
\end{aligned}
$$

\section{Next for}

\section{Output= uint8(Newimage $)$;}

In a similar manner, the color edges should be divided into several edges, according to the salient points, and obtain several independent edges for a single fragment. In a similar fashion, to extract the intersection of RGB colors from the surfaces of the fragments (as in [14]) for classifying the fragments into groups, this work relies on the same algorithm used for color feature extraction from the surfaces surrounding the edges of the fragments.

\section{E. Recognition using correlation coefficient function}

We briefly reviewed the fragment matching based on the edges of the curve feature. After obtaining the matrix of all coefficients and the colors intersecting all available edges, there should be a match between the fragments on the basis of such features. In general, since each fragment has four sides, it must recognize the sides that will be matched. In this case, the method uses the correlation coefficient, and nominates a pair of sides of fragments that have the highest relationship. This work use the correlation coefficient values because this produces better classification performance. Usually, the correlation coefficients are based on the nature and strength of the relationship between two variables, where its value ranges between the $(-1 \leq r \leq+1)$. The following formula represents the correlation coefficient between two variables, which are $x$ and $y[15]$.

$$
r_{x y}=\left(n \sum_{i=1}^{n} x_{i} y_{i}-\sum_{i=1}^{n} x_{i} \sum_{i=1}^{n} y_{i}\right) /\left(\sqrt{n \sum_{i=1}^{n} x_{i}^{2}-\left(\sum_{i=1}^{n} x_{i}\right)^{2}} \sqrt{n \sum_{i=1}^{n} y_{i}^{2}-\left(\sum_{i=1}^{n} y_{i}\right)^{2}}\right)
$$

Where $r_{x y}$ is the correlation between pair features of fragments $x$ and $y$, and $n$ is the number of coefficients in each polynomial equation.

Input four sets of features for first and second fragment.

$$
\begin{array}{r}
\text { Step1: For } k=1 \text { to } 4 \\
\text { For } p=1 \text { to } 4
\end{array}
$$

Calculate correlation coefficient between each side of fragment with other four sides according equation 3 .

Step2: Select highest relationship between the $|-1 \leq r \leq+1|$.

\section{End for}

Where $k$ is the coefficients of the first fragment, and $p$ represents the coefficients of the second fragment.

\section{EXPERIMENTAL SETUP}

After completing the theoretical aspect, an experiment was run upon the fragments obtained by a $2 \mathrm{D}$ camera. Therefore, a polynomial function and the intersection of colors were applied for reconstructing objects.

\section{A. Image acquisition and edges detection}

For initializing the proposed method, six 2D images were selected. Figure 1 represents the images that were loaded into memory.

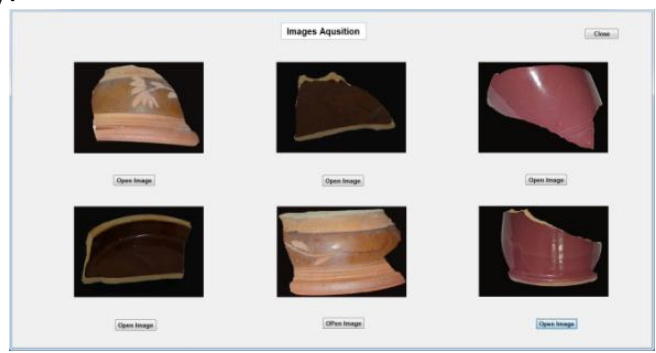

Fig. 1: Depicts six 2D images are loading

The procedure for separating each contour of the fragment according to the salient points was applied. In this experiment, each fragment includes the right side, left side, top side, and bottom side, as shown in Figure 2, which represents the results for the first group, include the first and fifth fragments. 

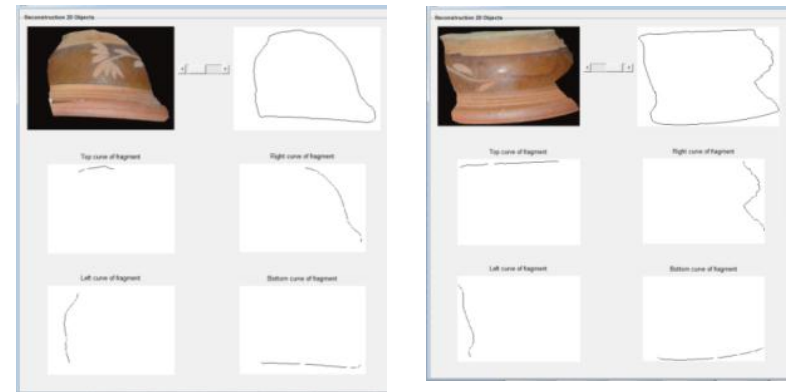

Fig. 2: Separate the contour for each fragment into four parts

Hence, each edge generated a matrix of $\left(x_{i}, y_{i}\right)$ points, so the result of this experiment is represented as a set of coordinates of points. Therefore, in order to obtain smooth curves, the values of the coordinates of the points were normalized to 0 and 1. Next, based on those points, a polynomial function of degree $n=6$ was created. Figure 3 shows the results.
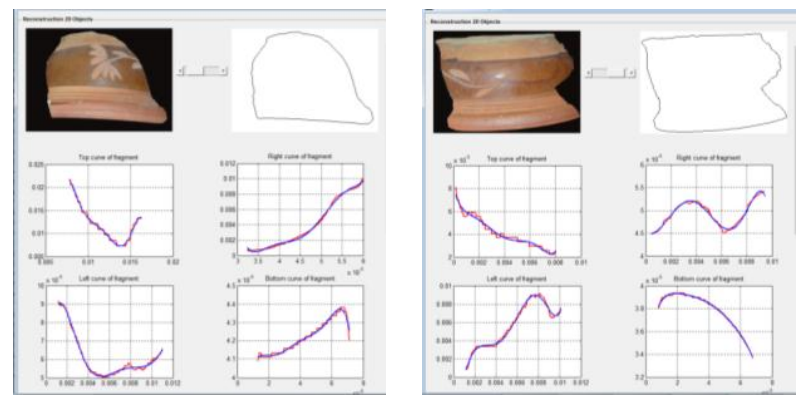

Fig. 3: Plotting the curves with a Polynomial function of six degree.

As a result, a vector of seven estimated coefficients was obtained to form the polynomial function for each curve, and the degree of each polynomial function is $6^{\text {th }}$. For each nonlinear value, the coefficients are in descending powers, so Equations 4 to 7 demonstrate the results for the four edges of the first fragment, while Equations 8 to 11 represent the results of the second fragment.
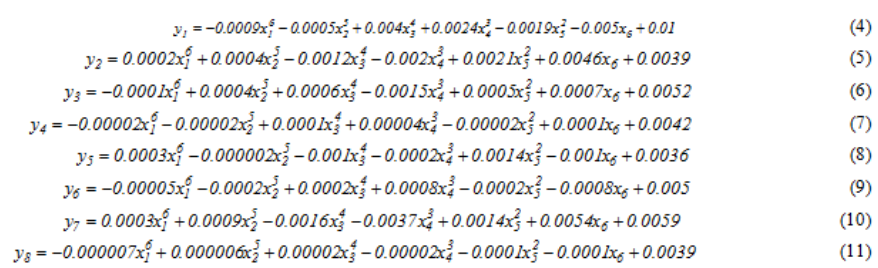

Based on this result, it can be deduced that the match has been achieved between a couple of the fragments by approximate similarity between the functions of curves. Therefore, the recognition technique should be applied to identify the pairs of edges of different fragments to be matched.

\section{B. Color feature extraction}

This work designed the model displayed in Figure 4a, which contains six buttons that enable the user to choose the group that should be reconstructed. Thus, in this experiment, we selected the first group, which represents the first and fifth fragments. Applying the first algorithm that allows the extraction of the strips of the edges shown in Figure $4 \mathrm{~b}$.
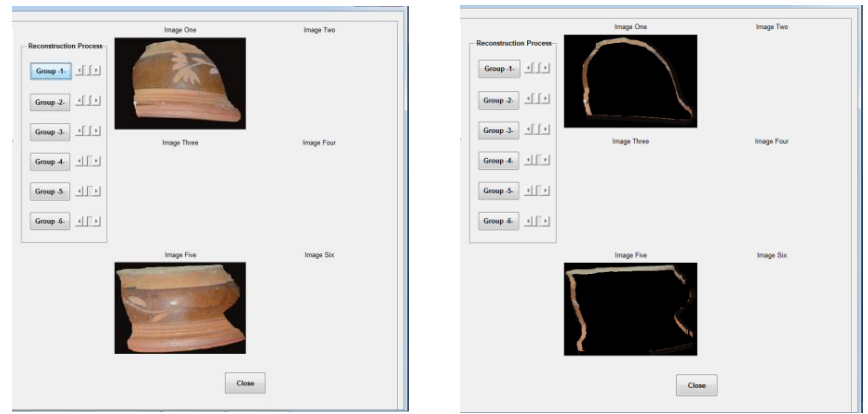

Fig. 4: a. Demonstrate the group should reconstructed; b. The strips of each fragment

Hence, as mentioned previously, each strip should be separated into sub-strips according to the salient points. Figure 5 shows the pairs of fragments that were separated into four parts for each one. In order to implement the recognition process and specify the pairs of sides that must match, we will calculate the intersection of colors between each edge of the first fragment with colors of all edges of other fragments. Table 1 represents the results.

TABLE I: RESUlT OF INTERSECTION BETWEEN FIRST AND SECOND FRAGMENTS

\begin{tabular}{lccccc}
\hline \hline & \multicolumn{5}{c}{ Second Fragment } \\
\cline { 2 - 6 } First Fragment & Edges & Top & Right & Left & Bottom \\
& Top & 47 & 5 & 18 & 0 \\
& Right & 16 & 50 & 62 & 21 \\
& Left & 8 & 32 & 37 & 19 \\
& Bottom & 1 & 2 & 0 & 0 \\
\hline \hline
\end{tabular}
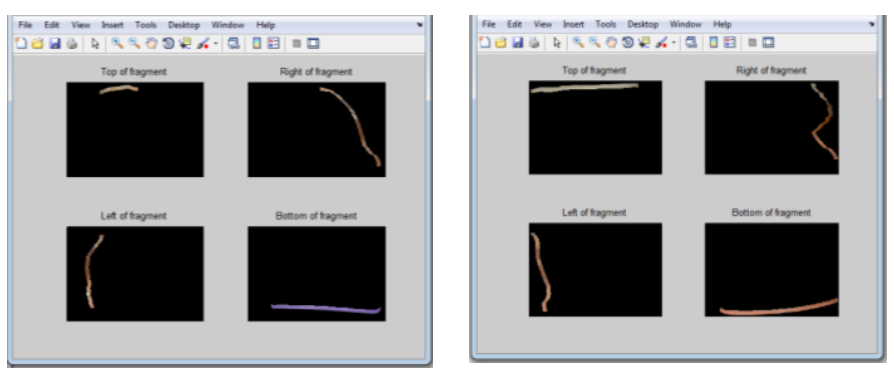

Fig. 5: Demonstrate two strips of fragments have been separated

\section{Apply recognition algorithm}

Finally, the correlation coefficient algorithm was applied using Tables 2, so as to obtain the relationship between two sets of coefficients $\left(y_{1} y_{5} \ldots y_{4} y_{8}\right)$. The results are shown in Table 3. As depicted in Figure 6, a strong relationship between a pairwise of two sides of pair fragments has been achieved in three positions. The highest value is 0.998742 , and these are between the upper edges of the vase, so this result was rejected because it is impossible for the pair of fragments to be matched from the upper part of the vase. Also, the intersection of colors in this region is low. The second highest value is 0.976825 , which represents the match between the left side of the first fragment and the right side of the second fragment. This result was accepted because both sides are compatible. In addition, the intersection of the colors in this region is the highest. The final result is shown in Figure 7. 
TABLE II: DEMONSTRATES THE COEFFICIENTS OF THE FIRST AND SECOND FRAGMENTS

\begin{tabular}{lcccccccc}
\hline \hline Coefficients & $y_{t}$ & $y_{2}$ & $y_{3}$ & $y_{4}$ & $y_{s}$ & $y_{s}$ & $y_{7}$ & $y_{s}$ \\
$a_{s}$ & -0.0009 & 0.0002 & -0.0001 & -0.00002 & 0.0003 & -0.00005 & 0.0003 & -0.00001 \\
$a_{5}$ & -0.0005 & 0.0004 & 0.0004 & -0.00002 & -0.000002 & -0.0002 & 0.001 & 0.00001 \\
$a_{t}$ & 0.004 & -0.0012 & 0.0006 & 0.0001 & -0.001 & 0.0002 & -0.002 & 0.00002 \\
$a_{3}$ & 0.0024 & -0.002 & -0.0015 & 0.00004 & -0.0002 & 0.0008 & -0.0037 & -0.00002 \\
$a_{2}$ & -0.0019 & 0.0021 & 0.0005 & -0.00002 & 0.0014 & -0.0002 & 0.0014 & -0.0001 \\
$a_{t}$ & -0.005 & 0.0046 & 0.0007 & 0.0001 & -0.001 & -0.0008 & 0.0054 & -0.0001 \\
$a_{5}$ & 0.01 & 0.004 & 0.0052 & 0.0042 & 0.0036 & 0.005 & 0.006 & 0.0039 \\
\hline \hline
\end{tabular}

TABLE III: DEMONSTRATES RESULT OF CORRELATION COEFFICIENT BETWEEN FIRST SECOND FRAGMENTS

\begin{tabular}{cccccc}
\hline \hline & \multicolumn{5}{c}{ Second Fragment } \\
\cline { 2 - 6 } & Edges & Top & Right & Left & Bottom \\
\hline \multirow{4}{*}{ First } & Top & 0.636103 & 0.902148 & 0.028618 & 0.816554 \\
Fragment & Right & 0.44433 & 0.287278 & 0.976825 & 0.476466 \\
& Left & 0.787359 & 0.829331 & 0.756745 & 0.928231 \\
& Bottom & 0.846384 & 0.968397 & 0.597387 & 0.998742 \\
\hline \hline
\end{tabular}

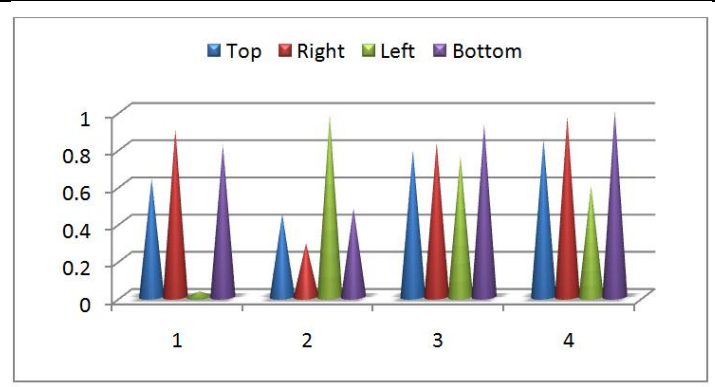

Fig. 6: Depicts the Correlation Coefficients Between First and Fifth Fragments

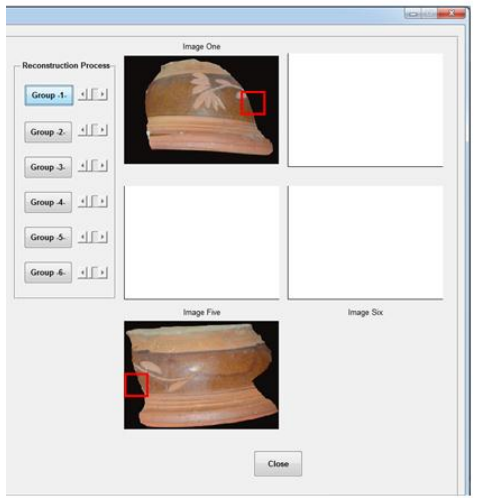

Fig. 7: Depicts the results of matching process.

\section{CONCLUSION}

In order to reconstruct the archaeological objects into their original form accurately, a robust method has been proposed that gives a high proportion of achievement to recognize the different sides of fragment pairs. This method is based on the edges and color features. After extracting the contours and dividing them according to the salient points, we applied a polynomial function and obtained the coefficients. Furthermore, we used the intersection of the colors near the edges of different fragments. This feature is important, because the broken edges of fragments remain to have the same colors. We conducted many experiments through using a dataset that consists of 30 fragments. After evaluation, we conclude that the proposed method achieves promising results, and is worthy to be studied further.

\section{ACKNOWLEDGEMENTS}

The researchers wish to thank Universiti Kebangsaan Malaysia (UKM) and Ministry of Higher Education, Malaysia for supporting this work by research grant DIP-2014-018 and FRGS/1/2014/ICT07/UKM/02/2.

\section{REFERENCES}

[1] T. Funkhouser, H. Shin, C. Toler-Franklin, A. García Castañeda, B. Brown, D. Dobkin, S. Rusinkiewicz, and T. Weyrich, "Learning how to match fresco fragments," In Proceedings of the Eurographics Area Track on Cultural Heritage, vol. 4, no. 2, pp.1-7, 2011.

[2] H. Shin, C. Doumas, T. Funkhouser, S. Rusinkiewicz, K. Steiglitz, A. Vlachopoulos, and T. Weyrich, "Analyzing and simulating fracture patterns of theran wall paintings," ACM Journal on Computing and Cultural Heritage, vol. 5, no. 3, pp.1-14, 2012.

http://dx.doi.org/10.1145/2362402.2362404

[3] V. Hristov, and G. Agre, "A Software system for classification of archaeological artefacts represented by 2D plans," Bulgarian Academy of Sciences Cybernetics and Information Technologies, vol. 13, no. 2, pp.82-96, 2013. http://dx.doi.org/10.2478/cait-2013-0017

[4] A. Castañeda, B. Brown, S. Rusinkiewicz, T. Funkhouser, and T. Weyrich, "Global consistency in the automatic assembly of fragmented artefacts," Proceeding of the 12th International Symposium on Virtual Reality. Archaeology And Cultural Heritage Vast, pp.73-80, 2011.

[5] K. Son, E.B. Almeida, and D.B. Cooper, "Axially symmetric 3D pots configuration system using axis of symmetry and break curve," IEEE Conference on Computer Vision and Pattern Recognition, pp.257-264, 2013. http://dx.doi.org/10.1109/cvpr.2013.40

[6] H. C. G. Leitao, and J. Stolfi, "A multiscale method for the reassembly of two dimensional fragmented objects," IEEE Transactions on Pattern Analysis and Machine Intelligence, vol. 24, no. 9, pp.1239-1251, 2002. http://dx.doi.org/10.1109/TPAMI.2002.1033215

[7] S. Yasuhara, S. Kato, and H. Itoh, H, "An earthenware reconstruction system considering color pattern and shape similarities," (English) Forma, vol. 19, no. 4, pp.413-425, 2004.

[8] B. Kimia, and H. Aras, "HINDSITE: A User-Interactive Framework for Fragment Assembly," IEEE Computer Society Conference on Computer Vision and Pattern Recognition Workshops, pp, 62-69, June 2010. http://dx.doi.org/10.1109/cvprw.2010.5543532

[9] C. Papaodysseus, P. Rousopoulos, D. Arabadjis, M. Exarhos, and M. Panagopoulos, "Reconstruction of c. 1650 B.C, fragmented wall paintings by exploitation of the thematic content," International Journal of Imaging, vol. 5, no.11, pp.1-7, 2011.

[10] S. Skembris, C. Papaodysseus, and E. Koukoutsis, "2D fragmented object reconstruction with the use of the chromatic and thematic content," Pattern Analysis and Application, vol.15, no.2, pp.133-146, 2012. http://dx.doi.org/10.1007/s10044-011-0251-9

[11] L.M. Rudner, and W.D. Schafer, What Teachers Need to Know about Assessment. National Education Association of the United States, pp.34-35, 2002.

[12] B. Sturmfels, Solving Systems of Polynomial Equations. 1st edition, American Mathematical Society, p. 152, 2002. http://dx.doi.org/10.1090/cbms/097

[13] N. A. Rasheed, and M. J. Nordin, "A Polynomial Function In The Automatic Reconstruction of Fragmented Objects," Journal of Computer Science, vol.10, no.11, pp. 2339-2348, 2014. http://dx.doi.org/10.3844/jcssp.2014.2339.2348

[14] N. A. Rasheed, and M. J. Nordin, "Archaeological Fragments Classification Based on RGB Color and Texture Features," Journal of Theoretical and Applied Information Technology, vol.76, no. 3, pp. 358$365,2015$. 
Int'I Journal of Computing, Communications \& Instrumentation Engg. (IJCCIE) Vol. 3, Issue 2 (2016) ISSN 2349-1469 EISSN 2349-1477

[15] G.R. Norman, and D.L. Streiner, Simple Regression and Correlation, Chapter Thirteenth of Biostatistics: The Bare Essentials, Third Edition, p.137, 2008. 